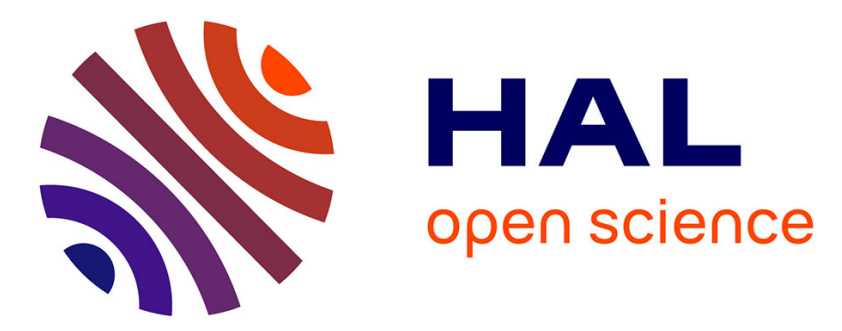

\title{
Optimizing the magnetic response of suspensions by tailoring the spatial distribution of the particle magnetic material
}

Laura Rodriguez Arco, Modesto Lopez-Lopez, Pavel Kuzhir, Georges Bossis, J.D.G Duran

\section{To cite this version:}

Laura Rodriguez Arco, Modesto Lopez-Lopez, Pavel Kuzhir, Georges Bossis, J.D.G Duran. Optimizing the magnetic response of suspensions by tailoring the spatial distribution of the particle magnetic material. ACS Applied Materials \& Interfaces, 2013, 5, pp.12143. 10.1021/am404035w . hal-00984536

\section{HAL Id: hal-00984536 \\ https://hal.science/hal-00984536}

Submitted on 28 Apr 2014

HAL is a multi-disciplinary open access archive for the deposit and dissemination of scientific research documents, whether they are published or not. The documents may come from teaching and research institutions in France or abroad, or from public or private research centers.
L'archive ouverte pluridisciplinaire HAL, est destinée au dépôt et à la diffusion de documents scientifiques de niveau recherche, publiés ou non, émanant des établissements d'enseignement et de recherche français ou étrangers, des laboratoires publics ou privés. 


\section{Optimizing the magnetic response of suspensions by tailoring the spatial distribution of the particle}

\section{magnetic material}

Laura Rodríguez-Arco, ${ }^{\dagger}$ Modesto T. López-López, ${ }^{\dagger}{ }^{*}$ Pavel Kuzhir, ${ }^{\star}$ Georges Bossis, ${ }^{\star}$ Juan D.G. Durán ${ }^{\dagger}$

${ }^{\dagger}$ Departamento de Física Aplicada, Facultad de Ciencias, Universidad de Granada, Campus de Fuentenueva, 18071 Granada, Spain.

tLaboratoire de Physique de la Matière Condensée, Université de Nice-Sophia Antipolis CNRS, Parc Valrose, 06108 Nice Cedex 2, Nice, France.

*Corresponding author. Email address: modesto@ugr.es

KEYWORDS: magnetic susceptibility; magnetic particles; colloids; rheology; magnetorheological fluids.

\section{ABSTRACT}

We report an experimental enhancement of the magnetic susceptibility of suspensions of particles, related to the spatial distribution of the magnetic phase in the particles. At low field the susceptibility of suspensions of nickel-coated diamagnetic spheres was approximately $75 \%$ higher than that of suspensions of solid nickel spheres with the same nickel content. This result 
was corroborated by magnetostatics theory and simulation. The distribution of the magnetic phase in a shell also led to an improvement of the field-induced rheological response of the suspensions.

\section{INTRODUCTION}

Tailoring the fundamental properties of matter has become a real possibility in recent decades thanks to the progress in nanotechnology and materials science and engineering. Actually, many synthetic materials with user-tunable properties have been already successfully commercialized..$^{1-4}$ Among the wide range of properties which can be easily manipulated, those related to magnetism have turned out to be powerful tools in many technological and biomedical applications. ${ }^{5}$ For example, sensitivity and efficiency of biosensors made of magnetic nanoparticles are improved by increasing the saturation magnetization of the nanoparticles. ${ }^{6}$ Remanence and coercitivity of magnetic nanoparticles used for hyperthermia applications can be tailored so that tissue heating is dominated either by relaxation processes -i.e. Néel or Brownian- or by hysteresis losses. ${ }^{7,8}$ An increase of the saturation magnetization of magnetic particles used as contrast agents in magnetic resonance imaging (MRI) also leads to higher effectiveness. $^{9-11}$ Similarly, an enhanced contrast in MRI applications can be achieved by increasing the magnetic susceptibility $\chi$ of the magnetic particles, because of the shortening effect of the spin-spin relaxation time. ${ }^{12,13}$

In addition to MRI, there are many other fields in which changes of $\chi$ are of practical interest: geophysics -e.g. paleomagnetism and environmental magnetism-, chemistry and physics - e.g. analytical chemistry, magnetic measurements- or magnetic field-responsive materials. ${ }^{14-16}$ As a matter of fact, many of these magnetic field-responsive materials - e.g. field-responsive fluids, foams, elastomers or gels- are built by incorporating colloidal ferromagnetic particles to a 
diamagnetic matrix. The magnetic susceptibility of the dispersed particles, and thus, the response to the field of the material, depends mainly on their composition -i.e. pure metals or ferrites- and on particle size -i.e. nano- vs. micron-size. For example, ferrofluids based on magnetite nanoparticles of relatively low $\chi$ display small changes of viscosity when a magnetic field is applied. ${ }^{16,17}$ On the other hand, magnetic fluids of iron microparticles of high $\chi$-also known as magnetorheological fluids-exhibit a much stronger change in their rheological, flow, properties in the presence of a field. ${ }^{16}$ Traditionally, the combination of different materials and/or sizes has offered a variety of responses to the applied magnetic field.

In this work we report a novel approach to enhance the magnetic susceptibility of particulate suspensions, based on the optimization of the spatial distribution of the magnetic material. In particular, we demonstrate both experimental and theoretically, that the magnetic susceptibility of a suspension of hollow magnetic spheres -i.e. diamagnetic-core-ferromagnetic-shell particles-, is enhanced in comparison to that of a suspension of solid ferromagnetic spheres with the same total amount of magnetic material. Such an improvement is expected to play a significant role on the magnetic field-induced rheology of the suspensions, which is also studied.

\section{EXPERIMENTAL}

We prepared two suspensions consisting of: (i) solid nickel spheres, average particle diameter (a.p.d.) of $10 \mu \mathrm{m}$ (Merck KgaA, Germany) and (ii) nickel-coated hollow ceramic spheres with a.p.d. of $12 \mu \mathrm{m}$ (Accumet Materials Co, USA), dispersed in mineral oil (Sigma Aldrich, USA) and stabilized by the addition of oleic acid (Sigma Aldrich, USA). The total particle volume fraction of (ii) was approx $40 \mathrm{vol} \%$. The one of (i) was adjusted so that both suspensions had the same amount of magnetic material -i.e. pure nickel. With this aim, we measured the magnetization $M$ of the prepared suspensions as a function of the magnetic field $H$ using a 
SQUID Quantum Design MPMS XL magnetometer. Then, we checked that both suspensions had the same saturation magnetization, which turned out to be $M_{s}=1.5 \mathrm{kA} \mathrm{m}^{-1}$, as shown in Fig. 1 . From this value we calculated the volume fraction of pure nickel by taking into account the saturation magnetization of bulk nickel $M_{s}=480 \mathrm{kA} \mathrm{m}^{-1} \cdot{ }^{14}$ By doing so, we obtained a volume fraction of pure nickel of $0.3 \mathrm{vol} \%$ for both suspensions.

We characterized the rheological behavior upon magnetic field application of both suspensions by means of a rheometer Thermo Haake RS 150. For this purpose, we used a parallel plate set specially adapted to apply magnetic fields in the direction parallel to the axis of the rheometer i.e. along the velocity gradient. The rheological measurements were conducted at 25, 60 and 85 ${ }^{\circ} \mathrm{C}$.

\section{RESULTS AND DISCUSSION}

As we mentioned above, both suspensions -i.e. consisting of solid nickel and nickel-coated spheres- had the same saturation magnetization and thus, the same content of pure nickel. In spite of this, we found important differences between the magnetization curves of the two samples -as shown in Fig. 1. As usual for suspensions of ferromagnetic particles both curves showed a first strong increase of $M$ at low fields -approx $H<200 \mathrm{kA} \mathrm{m}^{-1}$ - followed by a smoother one until $M_{S}$ was reached. Such a saturation -i.e. variation of $M$ of less than $1 \%^{-}$ appeared at lower fields for the solid nickel suspension, $H \sim 240 \mathrm{kA} \mathrm{m}^{-1}$, than for the nickelcoated one, $H \sim 320 \mathrm{kA} \mathrm{m}^{-1}$, as seen in Fig. 1. Besides, there were evident quantitative differences of susceptibility, $\chi=M / H$, for both suspensions in the region of low $H$ : the slope of the curve for the nickel-coated suspension was significantly higher than the one of solid nickel particles. Indeed, the calculation of $\chi$ in this range of fields revealed that such a difference of susceptibility was as big as $\sim 0.03-$ i.e. $75 \%-$ at the beginning of the curve -inset of Fig. 1 . 
Finally, at increasing fields, $\chi$ decreased for both suspensions until similar values were reached at the highest fields. But it was the great increase of $\chi$ at the lowest fields which indicated that a simple re-distribution of the magnetic material into a spherical shell led to a great enhancement of the magnetization degree of the suspension.

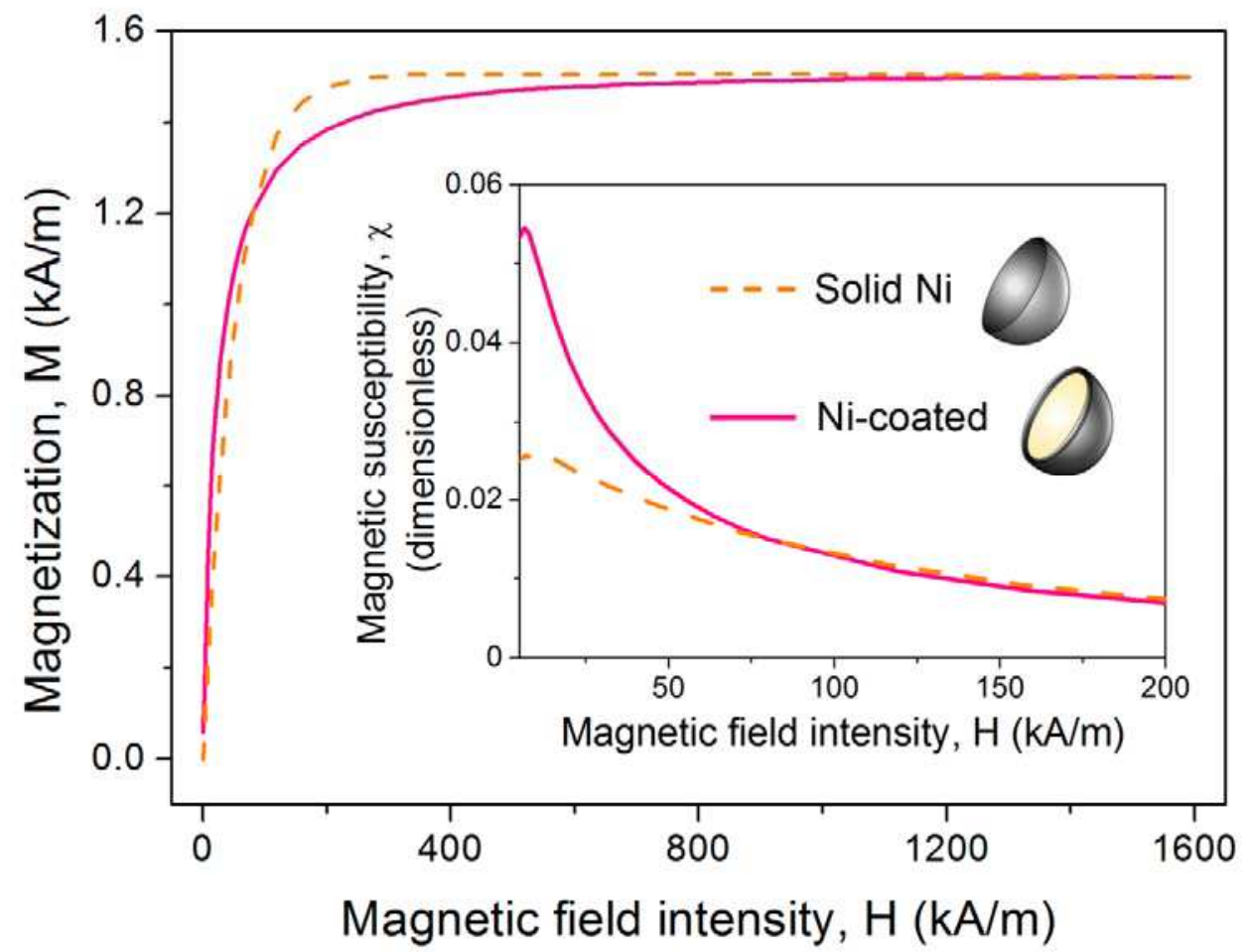

Figure 1. Experimental initial magnetization curves for both samples -i.e. consisting of solid nickel and nickel-coated spheres. Inset: magnetic susceptibility calculated from magnetization curves. The magnetic susceptibility was much higher for the nickel-coated suspension at low field in spite of having the same saturation magnetization -i.e. the same nickel content.

Let us now give a theoretical explanation to the experimentally observed trends by calculating the magnetic susceptibility of both suspensions. With this aim we first considered a dilute suspension of non-interacting, non-aggregated identical spheres of diamagnetic core $\left(\chi_{c} \approx 0\right)$ 
and magnetic shell $\left(\chi_{s}>0\right)$ dispersed in a diamagnetic matrix $\left(\chi_{m} \approx 0\right)$ and subjected to an external uniform field in the vertical direction $\vec{H}_{0}=H_{0} \hat{z}$. The radii of the core and outer surface of the shell were respectively $r_{c}$ and $r_{s}$, related by the parameter $p=\left(r_{c} / r_{s}\right)^{3}$. The average magnetization of the suspension $\langle\vec{M}\rangle$, only due to the magnetic shell, was calculated as follows:

$$
\langle\vec{M}\rangle=\frac{1}{V} \iiint_{V} \vec{M} d V=\frac{N_{p}}{V} \iiint_{V_{\text {stell }}} \vec{M}_{\text {shell }} d V_{\text {shell }}=\frac{N_{p} \chi_{s}}{V} \iiint_{V_{\text {shell }}} \vec{H} d V_{\text {shell }}
$$

In this equation $V$ was the suspension volume and $N_{p}$ the number of composite particles in the suspension. For simplification, we supposed in Eq. (1) that for each given value of the applied magnetic field the susceptibility was constant along the volume of the shell, i.e. its value did not change for the variations of the magnetic field within the shell. Otherwise it was not possible to solve Eq. (1) analytically. Note, however, that this approximation does not mean that the magnetic material was considered as linear since, as it will be shown below, the calculated susceptibility depended on the intensity of the magnetic field. The magnetic field was replaced by $\vec{H}=-\nabla \Psi_{s}$, with $\Psi_{s}$ being the magnetostatic potential in the shell:

$$
\langle\vec{M}\rangle=-\frac{N_{p} \chi_{s}}{V} \oiint_{S_{\text {stell }}} \Psi_{s} d \vec{S}_{\text {shell }}
$$

The shell potential was determined by using standard magnetostatics: ${ }^{18-20}$

$$
\Psi_{s}(r, \theta)=-H_{0}\left(A r-\frac{B p}{r^{2}}\right) \cos \theta \quad r_{c}<r<r_{s}
$$

Where $\theta$ and $r$ were, respectively, the polar angle and the radial position in spherical coordinates; $A$ and $B$ were constants determined by taking into account appropriate boundary conditions: ${ }^{18-20}$ 


$$
\begin{aligned}
& A=\frac{3}{\left(\mu_{s}+2\right)+2\left(\mu_{s}-1\right) \beta_{s} p} \\
& B=\frac{3 \beta_{s}}{\left(\mu_{s}+2\right)+2\left(\mu_{s}-1\right) \beta_{s} p}
\end{aligned}
$$

Where $\beta_{s}=\left(1-\mu_{s}\right) /\left(1+2 \mu_{s}\right)$ and $\mu_{s}$ was the magnetic permeability of the shell (i.e. nickel), related to the magnetic susceptibility by $\mu_{s}=\chi_{s}+1$.

The integral term in Eq. (2) was solved across the outer and the inner surfaces of the shell. Since the magnetic field was directed just along the $z$ axis and the shell was considered as an isotropic magnetic material, the magnetization vector had only one component which turned out to be:

$$
\left\langle M_{z}\right\rangle=\frac{N_{p} \chi_{s}}{V} \frac{4}{3} \pi r_{s}^{3} H_{0} A(1-p)
$$

Note that $\varphi=\left(N_{p} / V\right)(4 / 3) \pi r_{s}^{3}$ was the particle volume fraction of the suspension, whereas the term in brackets in Eq. (6) was the volume fraction of the shell in the whole composite particle. As a result, the volume fraction of magnetizable material in the suspension was given by $\varphi_{\text {mag }}=\varphi(1-p)$. The susceptibility of the whole suspension was calculated as:

$$
\chi_{\text {susp }}=\frac{\left\langle M_{z}\right\rangle}{H_{0}}=\chi_{s} A \varphi_{\text {mag }}
$$

Finally we substituted $A$ by Eq. (4) and introduced the magnetic contrast factor $\beta=\left(\mu_{s}-1\right) /\left(\mu_{s}+2\right)$, to obtain for the suspension of nickel-coated particles:

$$
\chi_{\text {susp }}=3 \varphi_{\text {mag }} \frac{\beta}{1+2 \beta \beta_{s} p}
$$


In the case of solid magnetic particles of radius $r$ dispersed in the same diamagnetic matrix, the mean magnetization of the suspension was calculated by considering the magnetic moment of each particle $\vec{m}$ :

$$
\langle\vec{M}\rangle=\frac{N_{p}}{V} \vec{m}=\frac{N_{p}}{V} 4 \pi \beta r^{3} \vec{H}_{0}
$$

Where, again, we supposed a system of non-interacting spheres. Since $\varphi_{\text {mag }}=\left(N_{p} / V\right)(4 / 3) \pi r^{3}$ in this second case, the magnetic susceptibility of the suspension of solid nickel particles turned out to be:

$$
\chi_{\text {susp }}=3 \varphi_{\text {mag }} \beta
$$

A comparison between Eqs. (8) and (10) revealed that the magnetic susceptibility of a suspension of hollow magnetic spheres should be enhanced with respect to the one of solid magnetic spheres for the same volume fraction of magnetic material $\varphi_{\text {mag }}$, since $\beta_{\mathrm{s}}<0$ in Eq. (8). Actually, for concentrated suspensions of high-permeability particles such a comparison is even more evident. If $\mu_{\mathrm{s}}>>1$, then $\beta \approx 1$ and $\beta_{s} \approx-1 / 2$, and the magnetic susceptibility of the suspension of hollow particles is larger than the one of the suspension of solid particles by a factor of $\varphi / \varphi_{\text {mag }}$.

Using Eqs. (8) and (10) we calculated the susceptibility of both suspensions. For this purpose, the values of $\mu_{s}$ as a function of $H$ for pure nickel were obtained from the materials library of FEMM software. $^{21}$ The theoretical curves followed the same tendency as the experimental results, that is, the susceptibility was higher for the suspension of nickel-coated particles -Fig. 2 . Actually, the theoretical difference between both samples was much higher than the experimental one. In particular, the calculated values of $\chi$ for the nickel-coated suspension were 
slightly higher than the experimental data -especially at the lowest fields- while in the case of the solid nickel suspension the situation was the opposite, i.e. the theory strongly underestimated $\chi$. However, and despite these quantitative differences the trends were identical, and consequently, basic magnetostatics allowed explaining the enhancement of susceptibility (see results in Fig. 2).

The discrepancies between experiments and theoretical calculations in Fig. 2 were likely due to the approximation of non-interacting and non-aggregated particles. Note that other phenomena, such as interparticle magnetic correlation and chain formation, which are known to play a significant role in the case of ferrofluids, ${ }^{22-24}$ should be ruled out in the present case due to the non-Brownian nature of the particles under study. In addition, the approximation made in Eq. (1) that the susceptibility of the magnetic material was constant along the particle shell might have been another source of discrepancy between theory and experiments.

A more precise estimation of the magnetic susceptibility of the suspensions requires the use of numerical methods. With this aim, we solved the magnetostatics problem by finite element method simulations (FEMM software ${ }^{21}$ ) for our two structured suspensions: chains of (i) solid nickel particles and (ii) nickel-coated particles. For this purpose, we considered an axisymmetric periodic cell of two particles (as shown in the inset of Fig. 2). Periodic boundary conditions were applied to both the upper and lower walls, and the internal magnetic field was imposed at the lateral (right) wall of the cell. The cell dimensions were adjusted so that the volume fractions of the different materials in the cells were the same as in the real suspensions (inset of Fig. 2). The results of the simulation are shown in Fig. 2.

The simulated results of the suspension magnetic susceptibilities were in good qualitative agreement with the experimental and theoretical ones, especially for the suspension of solid 
particles. Actually, in spite of some quantitative differences, the obtained trends were the same: the magnetic susceptibility was higher when the nickel phase was distributed in a thin spherical shell rather than in the case of a solid sphere (Fig. 2). The discrepancies with experiments were likely due to the real particle structures being different from the single-chain structures supposed here. $^{16}$

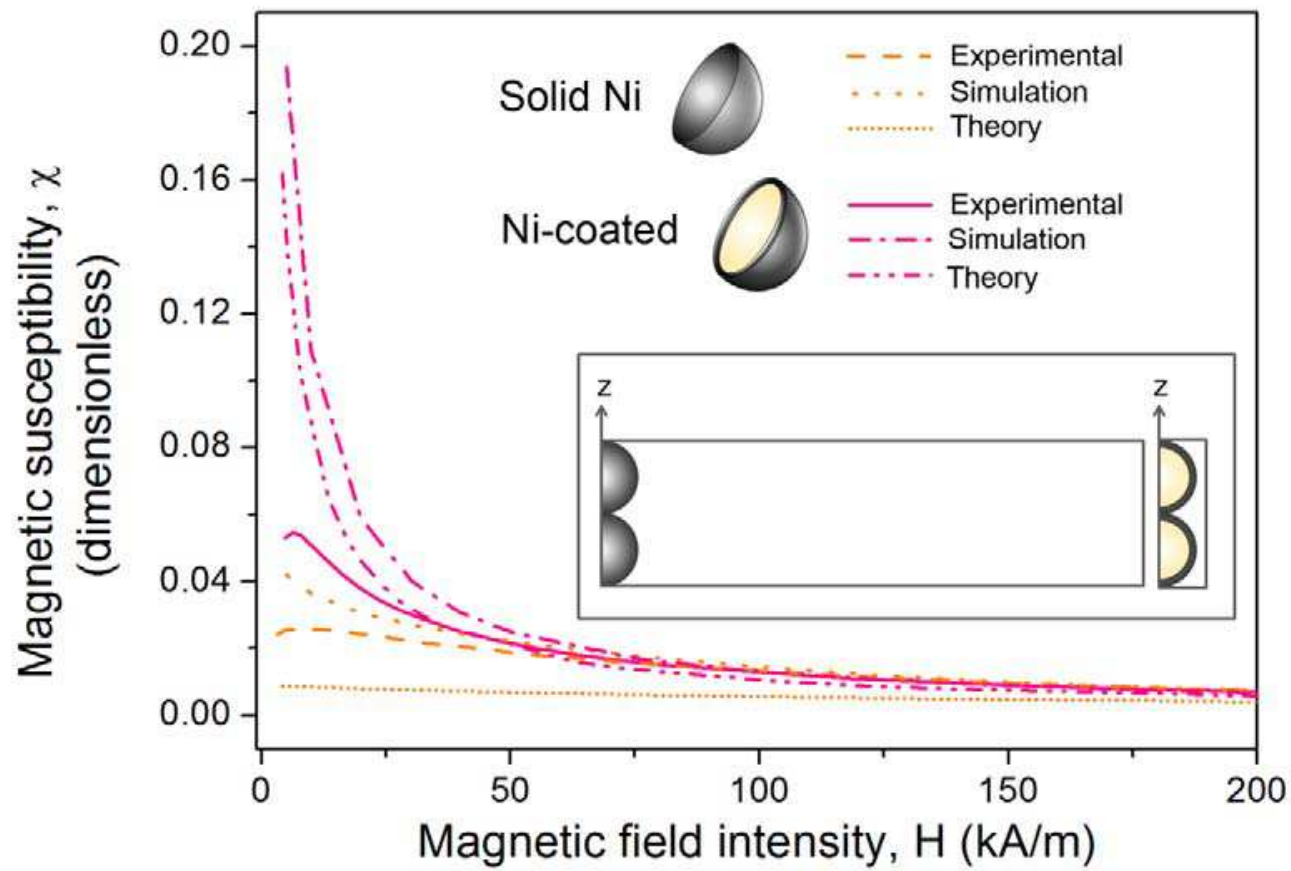

Figure 2. Results of theoretical calculations and FEMM simulation of the magnetic susceptibility for both suspensions. Experimental data are also plotted for comparison. Inset: schematic unit cells used in the simulation. Despite some quantitative differences, theory and simulation well reproduced the experimental trends: the magnetic susceptibility was higher for the nickel-coated suspension.

At this point it is worth mentioning that, as inferred from Eq. (8), the magnetic response of the nickel-coated suspension could be optimized by acting on parameter $p$ or, in other words, by acting on the thickness of the magnetic shell. Actually, parameter $p$ could ideally range from $p=$ 
0 -i.e solid nickel spheres- to $p=1-\frac{\varphi_{\operatorname{mag}}}{\varphi_{m}}$, where $\varphi_{m}$ was the maximum-packing volume fraction. In this range, the magnetic susceptibility of the nickel-coated suspension -i.e. Eq. (8) was a monotonic increasing function of parameter $p$, as shown in Fig.3. Such a result made evident that there is a progressive enhancement of the magnetic response as the magnetic shell became thinner.

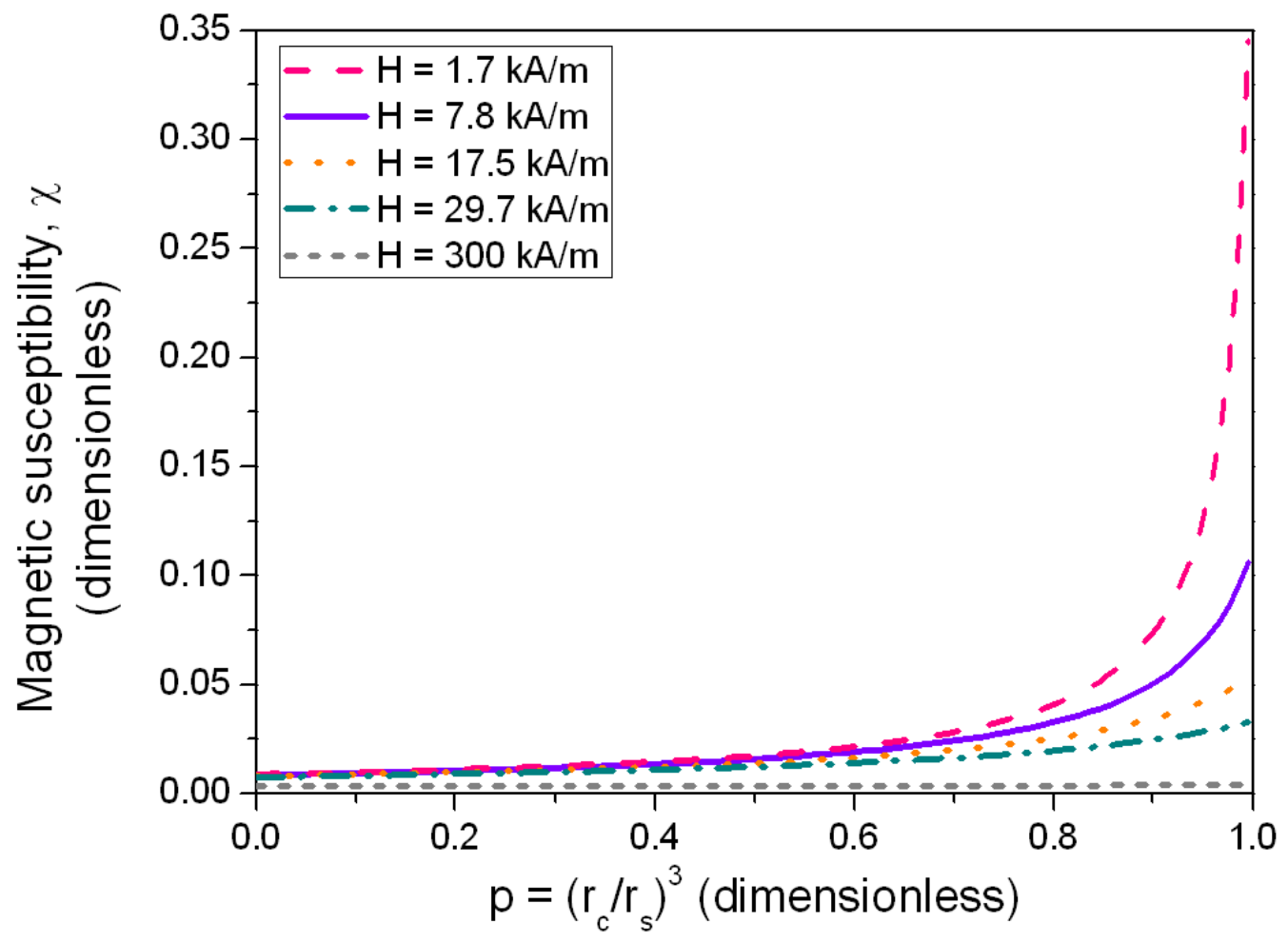

Figure 3. Magnetic susceptibility of the nickel-coated suspension as a function of parameter $p=\left(r_{c} / r_{s}\right)^{3}$ in the range from $p=0$ (solid nickel particles) to $p=1-\frac{\varphi_{m a g}}{\varphi_{m}}$, for different values of the magnetic field strength, $H$, obtained by means of Eq. (8). As seen, the susceptibility was a monotonic increasing function of $p$ and therefore, the thinner the magnetic shells, the stronger the magnetic response would be. 
The enhancement of the magnetizability of a suspension should lead to a better technological performance due to an improved response to external magnetic fields. As an example, we discuss here the effect on the rheological properties of the suspensions upon magnetic field application. As mentioned above, suspensions of magnetic micron-sized particles behave as field-responsive materials because they undergo changes in their rheological properties upon application of external fields. More specifically, in the absence of magnetic fields they usually follow Newton's law of viscosity - i.e. the shear stress $\sigma$ depends linearly on the shear rate $\dot{\gamma}$ in the form: $\sigma=\eta \dot{\gamma}$ , where $\eta$ is the viscosity. However, when an external field is applied, their viscosity strongly increases and a yield stress, $\sigma_{y}$, appears, below which the suspension does not flow. These fieldinduced rheological changes are due to the formation of magnetic particle structures in the field direction, which hinder the flow of the suspension. The formation and strength of the particle structures strongly depend on the degree of magnetization of the suspension, that is, on its magnetic susceptibility. ${ }^{16}$ Actually, theory and simulation previously indicated the possibility of enhancing the field-induced yield stress by using hollow magnetic spheres. ${ }^{25-27}$ However, no experimental evidence of such improvement has ever been published to the best of our knowledge. As a result, we decided to experimentally prove such a hypothesis by measuring the rheological properties of the suspensions. The yield stress was estimated by fitting the obtained rheograms $-\sigma$ vs $\dot{\gamma}$ graphs- to Bingham equation: $\sigma=\sigma_{y}+\eta \dot{\gamma}$. In order to analyze just the effect of the magnetic field we calculated the so-called increment of the yield stress with the magnetic field, $\Delta \sigma_{y}=\sigma_{y}(H)-\sigma_{y}(0)$ and plotted it against the external magnetic field $H$ in Fig. 4 -note that practically identical results were obtained when plotting it as a function of the internal magnetic field -not shown here for brevity- given the weak magnetizability of both suspensions (i.e. $\chi_{\text {susp }}<<1$ in both cases). ${ }^{14,28}$ 


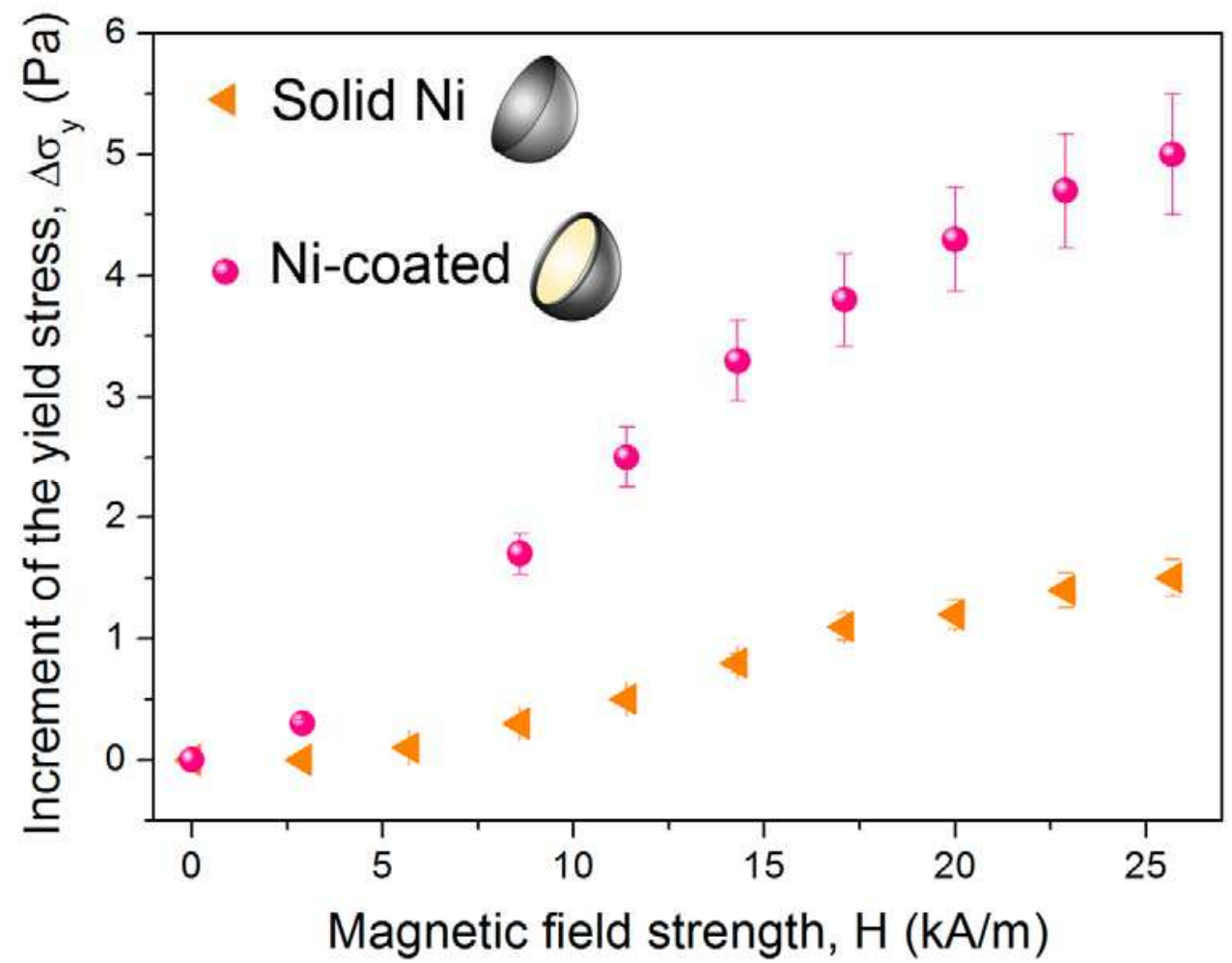

Figure 4. Increment of the yield stress as a function of the external magnetic field strength for both suspensions. This quantity was much higher for the nickel-coated sample for all the range of studied fields. Results were obtained from measurements at $25^{\circ} \mathrm{C}$.

A comparison between the $\Delta \sigma_{y}$ vs. $H$ curves for both suspensions revealed that, although they were similar in shape, there were big quantitative differences between them -Fig. 4. Both suspensions exhibited an increase of $\Delta \sigma_{y}$ with $H$, as a result of the strengthening of the fieldinduced particle chains with the field. However, $\Delta \sigma_{y}$ and its increase with $H$ were much higher for the nickel-coated sample, as a result of its stronger magnetization and an expected higher number of particle structures. Actually it was almost six times higher at the highest magnetic field -Fig. 4. Such a trend was maintained at higher temperatures, although a decrease of the 
yield stress with temperature was obtained -see figure S1 of Supporting Information- in agreement with previous works. ${ }^{29-31}$

The increase of the magnetic susceptibility for magnetically-coated particles made evident that it is possible to enhance the technological performance of magnetic field-responsive materials just by changing the distribution of the magnetic material in the dispersed particles. Hollow magnetic spheres are less dense than solid ones, which additionally reduces particle settling in suspensions, an important drawback which is usually present for these materials -see figure S2 of Supporting Information, in which a good stability over time can be observed for the nickelcoated sample, in contrast with the rapid sedimentation shown by the solid nickel one.

\section{CONCLUSIONS}

In summary, we have proved experimentally, theoretically and by finite element method simulation that the spatial distribution of magnetic material in colloidal particles affects the magnetic susceptibility of suspensions consisting of them. A suspension of nickel-coated hollow ceramic spheres (optimized system) gave rise to higher values of the initial susceptibility than a suspension of solid spheres (non-optimized system) with the same total volume fraction of nickel. In addition, we have theoretically shown that, for a given amount of magnetic material, the thinner the shell the higher the magnetic susceptibility is. Such an increase of susceptibility also improved the field-induced yield stress of the suspension, a parameter of practical interest in field-responsive fluids. Future works should reveal similar enhancements for other related applications. In addition, there is still work to be done in order to improve the technological performance of this kind of particles, the goal being the manufacture of cost-competitive, lowdensity, field-responsive materials, presenting an equivalent magnetic response and a large 
reduction of sedimentation and aggregation, which are always a concern for the applications of these materials.

\section{ASSOCIATED CONTENT}

Supporting Information. Discussion on the influence of the temperature on the yield stress and on the stability of the suspensions against gravitational particle settling. This material is available free of charge via the Internet at http://pubs.acs.org.

\section{ACKNOWLEDGMENT}

This work has been supported by Project P09-FQM-4787 (Junta de Andalucía, Spain). In addition, L. Rodríguez-Arco acknowledges financial support by Secretaría de Estado de Educación, Formación Profesional y Universidades (MECD, Spain) through its FPU and Estancias Breves programs. The authors also thank Prof. Dr. A. Zubarev for helpful discussion.

\section{REFERENCES}

(1) Ferrari, M. Nat. Rev. Cancer 2005, 5, 161-171.

(2) Vaddiraju, S.; Tomazos, I.; Burgess, D. J.; Jain, F. C.; Papadimitrakopoulos, F. Biosens. Bioelectron. 2010, 25, 1553-1565.

(3) Forshaw, M.; Stadler, R.; Crawley, D.; Nikolic, K. Nanotechnology 2004, 15, S220-S223.

(4) Schattling, P.; Jochum, F.; Theato, P. Multi-stimuli Responsive Polymers - the All-in-one Talents. Polym. Chem. [Online early access]. DOI: 10.1039/C3PY00880K. Published Online: Aug 7, 2013 http://pubs.rsc.org/en/content/articlelanding/2014/py/c3py00880k\#!divAbstract (accessed October 24, 2013). 
(5) Kolhatkar, A.G.; Jamison, A.C.; Litvinov, D.; Willson, R.C.; Lee, T.R. Int. J. Mol. Sci. 2013, 14, 15977-16009.

(6) Colombo, M.; Carregal-Romero, S.; Casula, M.F.; Gutierrez, L.; Morales, M.P.; Bohm, I.B.; Heverhagen, J.T.; Prosperi, D.; Parak, W.J. Chem. Soc. Rev. 2012, 41, 4306-4334.

(7) Khandhar, A.P.; Ferguson, M.R.; Simon, J.A.; Krishnan, K.M. J. Biomed. Mater. Res. 2011, $100 A, 728-737$.

(8) Chung, S.H.; Hoffmann, A.; Bader, S.D.; Liu, C.; Kay, B.; Makowski, L.; Chen, L. Appl. Phys. Lett. 2004, 85, 2971-2973.

(9) Yoo, D.; Lee, J.H.; Shin, T.H.; Cheon, J. Acc. Chem. Res. 2011, 44, 863-874.

(10) Lu, Z.; Yin, Y. Chem. Soc. Rev. 2012, 41, 6874-6887.

(11) Li, Y.; Wu, Y.; Luo, C.; Yang, F.; Qin, L.; Fu, T.; Wei, G.; Kang, X.; Wu, D. K, J. Mater. Chem. B 2013, 1, 4644-4654.

(12) Hadjipanayis, C.G.; Bonder, M. J.; Balakrishnan, S.; Wang, X.; Mao, H.; Hadjipanayis, G.C. Small 2008, 4, 1925-1929.

(13) Shokrollahi, H. Mater. Sci. Eng. C 2013, 33, 2476-2487.

(14) Jiles, D. Introduction to Magnetism and Magnetic Materials; Chapman and Hall Publishers: London and New York, 1994; pp 3-61 and pp 89-109.

(15) Mulay, L.N.; Mulay, I.L. Anal. Chem. 1980, 52, 199R-214R.

(16) Bossis, G. ; Volkova, O. ; Lacis, S.; Meunier, A. Lect. Notes Phys. 2002, 594, 201-230. 
(17) Rosensweig, R.E. Ferrohydrodynamics; Cambridge University Press: New York, 1985; pp 54-67.

(18) Jones, T.B. Electromechanics of Particles; Cambridge University Press: New York, 1995; pp 227-235.

(19) Landau, L.D.; Lifshitz, E.M. Electrodynamics of Continuous Media; Pergamon: New York, 1984; pp 113-165.

(20) Yu, K.W.; Yuen, K.P. J. Phys. Condens. Matter 1996, 8, 11327-11336.

(21) Finite Element Method Magnetics, version 4.2; software for solving magnetostatics problems by finite element methods; Meeker, D. C.: USA, 2009.

(22) Ivanov, A.O.; Kuznetsova, O.B. Phys. Rev. E 2001, 64, 041405.

(23) Mendelev, V.S.; Ivanov, A.O. Phys. Rev. E 2004, 70, 051502.

(24) Klokkenburg, M.; Erné, B.H.; Mendelev, V.S.; Ivanov, A.O. J. Phys. Condens. Matter 2008, 20, 2041113.

(25) Bossis, G.; Lemaire, E. J. Rheol. 1991, 35, 1345-1354.

(26) Lemaire, E. Electro- and Magnetorheological Suspensions. Ph.D. Thesis, University of Nice-Sophia Antipolis, Nice, France, 1992.

(27) Rodríguez-Arco, L.; López-López, M.T.; Kuzhir, P.; Durán, J.D.G. Soft Matter 2013, 9 , $5726-5737$. 
(28) López-López, M.T.; Kuzhir, P.; Caballero-Hernández, J.; Rodríguez-Arco, L.; Durán, J.D.G.; Bossis, G. J. Rheol. 2012, 56, 1209-1224.

(29) Weiss, K.D.; Duclos, T.G. Int. J. Mod. Phys. B 1994, 8, 3015-3032.

(30) Li, W.H.; Chen, G.; Yeo, S.H.; Du, H. Int. J. Mod. Phys. B 2002, 16, 2725-2731.

(31) Guerrero-Sanchez, C.; Ortiz-Alvarado, A.; Schubert, U.S. J. Phys. Conf. Ser. 2009, 149, 012052. 